\title{
Brain magnetic resonance imaging and multimodal evoked potentials in benign and secondary progressive multiple sclerosis
}

\author{
Massimo Filippi, Adriana Campi, Silvia Mammi, Vittorio Martinelli, Tiziana Locatelli, \\ Giuseppe Scotti, Stefano Amadio, Nicola Canal, Giancarlo Comi
}

\begin{abstract}
Brain MRI and multimodal evoked potentials (EPs) were obtained for 13 patients with benign multiple sclerosis and 13 patients with secondary progressive multiple sclerosis, matched for age and duration of the disease, to investigate the nature of the disability in multiple sclerosis. Patients with secondary progressive multiple sclerosis had significantly greater lesion loads for five of seven periventricular regions and for three of nine regions separate from the ventricles. Patients with secondary progressive multiple sclerosis also had more severe infratentorial atrophy scores $(p=$ $0 \cdot 04)$, whereas there were no differences between the two groups in number and extent of enhancing lesions.
\end{abstract}

The frequencies were significantly higher and severities greater for multimodal EP abnormalities of all the modalities in patients with secondary progressive multiple sclerosis. At least one EP component was absent in 12 $(92 \%)$ patients with secondary progressive multiple sclerosis but in only one patient $(8 \%)$ with benign multiple sclerosis $(p<0.001)$. There was neurophysiological evidence for cervical cord involvement in eight (61\%) patients with secondary progressive multiple sclerosis and in one with benign multiple sclerosis (p $<0.01$ ).

These data indicate that the total amount of lesions, the distribution, and the nature of the pathological process might all account for the development of disability in multiple sclerosis.

Scientific Institute Ospedale San Raffaele, University of Milan, Italy

Department of

Neurology

M Filippi

$S$ Mammi

V Martinelli

T Locatelli

$S$ Amadio

N Canal

G Comi

Department of Neuroradiology A Campi

Correspondence to: Professor Giancarlo Comi Department of Neurology, Scientific Institute Ospedale San Raffaele, Via Olgettina, 60, 20132 Milan, Italy.

Received 28 March 1994 Accepted 5 August 1994
There are several biological explanations for these clinicopathological paradoxes, ${ }^{45}$ such as the pathological characteristics of chronic lesions, the severity of microscopic changes in the normal appearing white matter, the distribution of the lesions, the capacity for remyelination, and the degree of recovery of conduction in persistently demyelinated axons. Some methodological aspects must also be taken into account, however, when considering the results of these studies. In the study by Koopmans et $a l,{ }^{3}$ brain lesion loads of patients with benign multiple sclerosis were compared with those of patients with both secondary and primary progressive multiple sclerosis, but patients with primary progressive multiple sclerosis characteristically have very mild brain involvement. ${ }^{2}$ In the study by Thompson et al MRI lesion loads of patients with secondary progressive multiple sclerosis were compared separately with those of patients with benign multiple sclerosis. The benign group, however, had durations of the disease about three-fold longer. Therefore, it is possible that a faster rate of lesion development might have accounted for different degrees of disability by interfering with mechanisms of repair. ${ }^{6}$

Also, standard T2 weighted MR sequences give information only about the site and the extent of multiple sclerosis lesions, not allowing any evaluation of the pathological characteristics of the process. Furthermore, we still do not know the relation between the brain lesion load and the spinal cord lesion load in multiple sclerosis, and it is likely that most of the disability in multiple sclerosis might be explained by spinal cord damage. Multimodal evoked potentials (EPs), although they exclusively explore specific nerve pathways, can be used to evaluate indirectly the amount of demyelination and axonal loss in the CNS of patients with multiple sclerosis ${ }^{7-9}$ by exploring the functional consequences of the disease process. Multimodal evoked potentials also provide information about CNS structures, such as the spinal cord and the optic nerves, which are not as well visualised by MRI, but which are of great importance in causing disability in multiple sclerosis when damaged.

The aim of this study was to achieve a better definition of the nature of disability in multiple sclerosis. For this, we compared brain MRI and multimodal EP findings of two groups of patients with multiple sclerosis with different patterns of disease evolution-that is, benign and secondary progressive multiple sclerosis matched for age and duration of the disease. 


\section{Patients and methods \\ PATIENTS}

Twenty six patients with clinically definite multiple sclerosis ${ }^{10}$ volunteered for the study and provided informed consent. Patients were selected by two of us (SM and VM) from the multiple sclerosis population attending the multiple sclerosis centre of the Scientific Institute, Ospedale San Raffaele, University of Milan. Thirteen patients had benign and 13 had secondary progressive multiple sclerosis. The two groups of patients were matched for age and duration of the disease. Patients with benign disease were defined as those having a Kurtzke expanded disability status scale $(E D S S)^{11}$ score of 3 or less after a disease duration of greater than 10 years. Patients with secondary progressive multiple sclerosis had had an initial relapsing and remitting phase but had developed progressive disability over at least the past six months. To be included, their EDSS scores had to be at least 5. All patients had CSF oligoclonal bands.

Patients were not included in the study if they had had a clinical relapse in the previous three months or had been treated with steroids or psychotropic drugs. Previous immunosuppressive treatments had to have ended at least two years before starting this study. All patients underwent brain MRI. Visual EPs (VEPs) were obtained for 25 patients (the remaining patient had secondary progressive multiple sclerosis), brainstem auditory EPs (BAEPs) for 23 (the remaining three patients had secondary progressive multiple sclerosis), left and right median somatosensory EPs (mSEPs) for 24 (the remaining two patients had secondary progressive multiple sclerosis), left and right tibial somatosensory EPs for 24 (one of the remaining two patients had benign multiple sclerosis and the other secondary progressive multiple sclerosis) and, left and right upper and lower limb motor EPs (MEPs) for 23 (one of the remaining three patients had benign multiple sclerosis and the other two secondary progressive multiple sclerosis). The tests were all performed within a week.

\section{MAGNETIC RESONANCE IMAGING}

T2 weighted MRI of the brain was performed with a Siemens system operating at 1.5 Tesla (SE 2400/25-90, 5-mm contiguous axial and 4 mm sagittal slices, $256 \times 256$ image matrix). Gadolinium-diethylenetriaminepenta-acetic acid (Gd-DPTA) was injected intravenously $(0.1 \mathrm{mM} / \mathrm{kg}$ ) and $\mathrm{T} 1$ weighted scans (SE 700/17, 5-mm axial contiguous slices, image matrix $192 \times 256$ ) were obtained after repositioning the patient (delay five to seven minutes). Scans were examined consensually by three of us (MF, AC, and GS), unaware of patients' disease pattern. Enhancing and nonenhancing lesions were counted and sized for 16 anatomically defined locations (seven periventricular and nine separate from the ventricles). An arbitrary scoring system weighted for lesion size was used to estimate total and regional lesion loads: 1 point was given for each lesion with a diameter 1-5 mm,
2 points for one $6-10 \mathrm{~mm}$, and 3 points for one over $10 \mathrm{~mm}$ in size. Confluent lesions scored one extra point. Supratentorial, infratentorial, and corpus callosum atrophy were scored as follows: score 0: normal size; score 1: mild atrophy; score 2: moderate atrophy; score 3: severe atrophy.

\section{NEUROPHYSIOLOGICAL TESTS}

For pattern reversal VEPs a black and white checkerboard pattern on a square TV screen, reversing every $0.7 \mathrm{~ms}$, was employed. The entire stimulating field was $11^{\circ}$; both $30^{\prime}$ and $15^{\prime}$ check size were used. The contrast was kept constant at $50 \%$. The cortical responses were recorded from $\mathrm{Oz}$ referred to $\mathrm{Cz}$. Analysis time was $250 \mathrm{~ms}$ and the bandpass was 1-100 Hz. The VEPs were considered abnormal if the P100 latencies exceeded 121 ms for check size $30^{\prime}$ or $125.5 \mathrm{~ms}$ for check size $15^{\prime}(2 \cdot 5 \mathrm{SD}$ from the normal mean values used in our laboratory and obtained from 60 age and sex matched controls) or if the cortical responses were absent or of low amplitude $(<2.5 \mathrm{mcV})$. We considered asymmetry greater than $8 \mathrm{~ms}$ in latency or greater than $50 \%$ in amplitude as significant.

The BAEPs were recorded in a quiet room in the usual manner with the patient in a supine position. Monoaural square wave click stimuli (duration $0.1 \mathrm{~ms}$ ) were delivered by a shielded earphone at $100 \mathrm{~dB}$ SPL, rate $21 \mathrm{~Hz}$. The first $15 \mathrm{~ms}$ after stimuli were analysed. The EPs after rarefaction and condensation clicks were recorded simultaneously and alternating-phase BAEPs were summed up off line from the rarefaction and condensation BAEP. Two trials of 2000 sweeps were averaged for each ear. Brain activity was recorded on two channels $(\mathrm{Cz}-\mathrm{A} 1, \mathrm{Cz}-\mathrm{A} 2)$ with a bandwidth of 1-2000 Hz. The potential was considered abnormal when waves III or V were absent or had increased latencies or the interpeak latencies I-III, III-V, or I-V were increased (values $2.5 \mathrm{SD}$ more than the mean values obtained for 60 sex and age matched controls).

For mSEPs, left and right median nerves were stimulated at the wrist with electrical square wave pulses with a duration of $0.1 \mathrm{~ms}$. The stimulation rate was $3.9 \mathrm{~Hz}$ and the stimulus intensity just above the motor threshold. Two averages, 1000 sweeps each, were collected, with the analysis time $50 \mathrm{~ms}$ and bandpass 5-2000 Hz. Recording electrodes were placed over Erb, Cv7, Fpz, and C3' or C4'. For tSEPs, left and right tibial nerves were stimulated at the ankles with the same protocol used for mSEPs. Recording electrodes were placed over $\mathrm{Cz}^{\prime}$ referred to $\mathrm{Fpz}$ for cortical evoked responses and over D12 referred to L3 and to anterior iliac spine for spinal evoked responses. Analysis time was $100 \mathrm{~ms}$ and bandpass 5-2000 Hz. The SEPs were considered abnormal when spinal cord (N13 for mSEPs), brainstem (P14 for mSEPs), or cortical (N20 for mSEPs and P40 for tSEPs) potentials were absent in patients with preserved peripheral conduction (normal P11 for mSEPs and N24 for tSEPs) or when increased interpeak latencies (N13-P14, 
P11-P14, N13-N20 for mSEPs and N24-P40 for tSEPs) were recorded (values exceeding the mean $+2.5 \mathrm{SD}$ obtained from 60 sex and age matched healthy controls).

At least two trials of both EPs were performed to see the reproducibility of the traces. The mSEPs and tSEPs were obtained by the Nicolet Pathfinder II, BAEPs and VEPs by the Amplaid MK7 apparatus.

Magnetic stimulation of the motor cortex was performed with a Cadwell MS10 machine. The coil was placed tangentially to the scalp, with its centre over the vertex; its position was slightly altered for each case to obtain the largest MEP from the target muscles, the abductor pollicis brevis and the abductor hallucis. Patients were asked to slightly contract target muscles (about $20 \%$ of maximum voluntary effort) to facilitate cortical motor responses. Spinal roots were stimulated by placing the coil over C6-C7 and L4-L5 spaces, with recordings from the same muscles. Stimulus intensity was $100 \%$ of the maximum of the stimulator for all subjects. Total motor conduction time (TMCT) and peripheral motor conduction time (PMCT) were obtained. Central motor conduction time was measured as the difference between TMCT and PMCT. The MEPs were considered abnormal when absent after cortical stimulation but present after peripheral stimu-

Table 1 Clinical description of patients with benign and secondary progressive multiple sclerosis

\begin{tabular}{llll}
\hline & $\begin{array}{l}\text { Benign multiple } \\
\text { sclerosis }\end{array}$ & $\begin{array}{l}\text { Secondary progressive } \\
\text { multiple sclerosis }\end{array}$ & p Value \\
\hline Mean (SD) age at onset (y) & $39(7 \cdot 3)$ & $40(6 \cdot 4)$ & NS \\
Mean (SD) duration of the disease (y) & $14 \cdot 2(4 \cdot 9)$ & $14 \cdot 1(4 \cdot 9)$ & NS \\
Mean (SD) Kurtzke EDSS & $1 \cdot 9(0 \cdot 6)$ & $6 \cdot 1(0 \cdot 6)$ & $0 \cdot 0001$ \\
\hline
\end{tabular}

«Student's $t$ test.

Table 2 Brain MRI lesion loads in benign and secondary progressive multiple sclerosis

\begin{tabular}{llll}
\hline & $\begin{array}{l}\text { Benign multiple } \\
\text { sclerosis } \\
\text { Median (range) }\end{array}$ & $\begin{array}{l}\text { Secondary progressive } \\
\text { multiple sclerosis } \\
\text { Median (range) }\end{array}$ & p Value \\
\hline Total lesion load & $28(6-89)$ & $57(25-117)$ & 0.03 \\
Periventricular lesion load & $13(0-43)$ & $33(11-62)$ & 0.01 \\
Non-periventricular lesion load & $15(2-56)$ & $26(13-60)$ & NS \\
Infratentorial lesion load & $1(0-10)$ & $7(3-19)$ & 0.01 \\
\hline
\end{tabular}

${ }^{\star}$ Mann-Whitney test.

\section{Total brain MRI lesion} loads in patients with benign and secondary progressive multiple sclerosis.

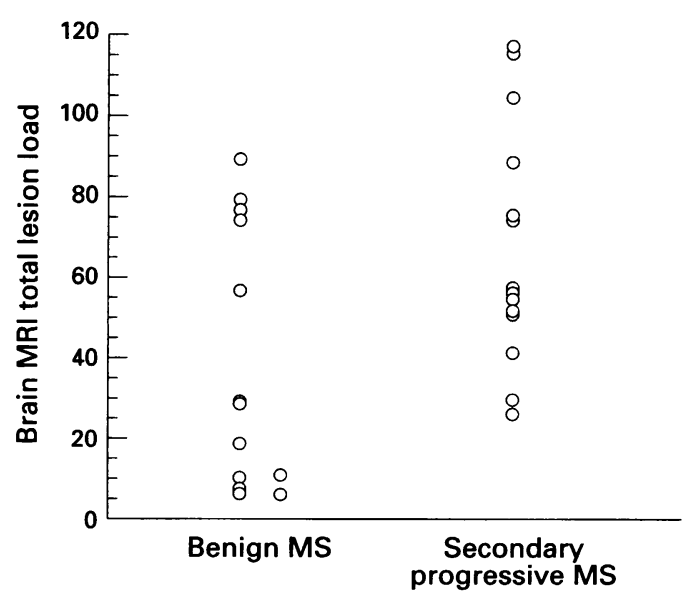

lation or when the latencies were greater than the mean normal values $+2.5 \mathrm{SD}$. The control group consisted of 40 sex and age matched healthy subjects.

The severity of the neurophysiological abnormalities was scored for each modality as follows: normal EP score 0; every other EP abnormality except the absence of one of the main waves, score 1 ; absence of one or more of the main waves score 2 .

\section{STATISTICAL ANALYSIS}

The clinical variables were compared by the two tailed Student's $t$ test for paired data. The Mann-Whitney test (as the values were not distributed normally) was used to compare (a) brain MRI total and regional lesion loads, (b) supratentorial, infratentorial, and corpus callosum atrophy scores, (c) number and extent of enhancing lesions, and (d) the severity of EP abnormalities (for each modality the worst score was used in the analysis). The confluence of MRI lesions, the frequency of EP abnormalities, and the presence of neurophysiological abnormalities indicating cervical cord involvement were compared by $\chi^{2}$ test.

\section{Results}

CLINICAL DATA (TABLE 1)

According to the protocol, the patients with benign and secondary progressive multiple sclerosis had similar mean ages and durations of the disease, whereas they had significantly different mean EDSS scores $(p<0.0001)$.

\section{BRAIN MRI}

Patients with benign multiple sclerosis had significantly lower total lesion loads than patients with secondary progressive multiple sclerosis $(p=0.03 ;$ table 2$)$. Five patients with benign multiple sclerosis $(38 \%)$ had total lesion loads of 10 or less, whereas the lowest value in patients with secondary progressive multiple sclerosis was 25 (figure). Confluence of lesions was seen in $10(77 \%)$ patients with secondary progressive multiple sclerosis and in only three $(23 \%)$ patients with benign multiple sclerosis $(p=0.02)$. In three of 13 pairs (23\%) the MR detected lesion load was, however, greater in patients with benign multiple sclerosis.

Patients with secondary progressive multiple sclerosis had significantly higher scores for five of seven periventricular regions (frontal and occipital horns, trigones, third and fourth ventricles; table 3 ) and for three of nine regions separate from the ventricles (brainstem, basal ganglia, and corpus callosum; table 4). These differences in regional lesion loads accounted for overall significant differences in the scores for periventricular $(p=$ $0.01)$ and infratentorial $(p=0.01)$ regions (table 2).

Six patients with benign multiple sclerosis had 10 enhancing lesions (eight with a diameter smaller than $5 \mathrm{~mm}$ and two larger than 10 $\mathrm{mm}$ ) and six patients with secondary progressive multiple sclerosis had 20 (10 with a diameter smaller than $5 \mathrm{~mm}$, six with a diameter 
Table 3 Brain MRI lesion loads for periventricular regions

\begin{tabular}{llll}
\hline & $\begin{array}{l}\text { Benign multiple } \\
\text { sclerosis } \\
\text { Median (range) }\end{array}$ & $\begin{array}{l}\text { Secondary progressive } \\
\text { multiple sclerosis } \\
\text { Median (range) }\end{array}$ & p Value* \\
\hline Body of lateral ventricles & $7(0-23)$ & $16(4-25)$ & NS \\
Frontal horns & $0(0-6)$ & $6(1-14)$ & 0.002 \\
Trigones & $2(0-6)$ & $6(0-10)$ & 0.0007 \\
Occipital horns & $2(0-9)$ & $6(0-13)$ & 0.03 \\
Temporal horns & $0(0-6)$ & $2(0-6)$ & NS \\
Third ventricle & $0(0-0)$ & $0(0-5)$ & 0.01 \\
Fourth ventricle & $0(0-1)$ & $1(0-6)$ & 0.001 \\
\hline
\end{tabular}

«Mann-Whitney test.

Table 4 Brain MRI lesion loads for non-periventricular regions

\begin{tabular}{llll}
\hline & $\begin{array}{l}\text { Benign multiple } \\
\text { sclerosis } \\
\text { Median (range) }\end{array}$ & $\begin{array}{l}\text { Secondary progressive } \\
\text { multiple sclerosis } \\
\text { Median (range) }\end{array}$ & p Value* \\
\hline Frontal lobe & $9(0-24)$ & $8(2-26)$ & NS \\
Parietal lobe & $2(0-11)$ & $3(0-10)$ & NS \\
Occipital lobe & $0(0-4)$ & $1(0-6)$ & NS \\
Temporal lobe & $1(0-3)$ & $2(0-7)$ & 0.01 \\
Corpus callosum & $0(0-5)$ & $2(0-6)$ & 0.005 \\
Basal ganglia & $0(0-2)$ & $2(0-7)$ & NS \\
Internal capsules & $2(0-6)$ & $3(0-7)$ & 0.02 \\
Brainstem & $0(0-7)$ & $4(1-7)$ & NS \\
\hline Cerebellum & $1(0-5)$ & $1(0-6)$ & \\
\hline
\end{tabular}

${ }^{\star}$ Mann-Whitney test.

between 6 and $10 \mathrm{~mm}$, and four with a diameter larger than $10 \mathrm{~mm}$ ). This difference was not statistically significant $(p=0 \cdot 07)$.

There were no differences in the severity of supratentorial and corpus callosum atrophy in the two groups, but patients with secondary progressive multiple sclerosis had more severe infratentorial atrophy than patients with benign multiple sclerosis $(p=0.04)$.

\section{MULTIMODAL EVOKED POTENTIALS}

The frequencies were significantly higher and the bilaterality of multimodal EP abnormalities significantly greater for all the modalities (except the frequency of abnormalities for VEPs) in patients with secondary progressive multiple sclerosis, with the greatest significances for SEPs and MEPs (table 5).

The severity of the abnormalities-that is, the absence of one or more of the main components of the evoked responses-was also greater in patients with secondary progressive multiple sclerosis, with the following $p$ values: VEPs $p=0.02$; BAEPs $p=0.005$; mSEPs $p$ $=0.001$; tSEPs $\mathrm{p}<0.0001$; upper MEPs $\mathrm{p}<$ 0.0001 ; and lower limb MEPs $p=0.005$. At least one EP component was absent in 12 (92\%) patients with secondary progressive

Table 5 Frequencies of abnormality of multimodal EPs

\begin{tabular}{llll}
\hline & $\begin{array}{l}\text { Benign multiple } \\
\text { sclerosis } \\
\text { No }(\%)\end{array}$ & $\begin{array}{l}\text { Secondary progressive } \\
\text { multiple sclerosis } \\
\text { No }(\%)\end{array}$ & p Value* \\
\hline VEPs & $6 / 13(46)$ & $10 / 12(83)$ & NS \\
Bilateral abnormalities & $4 / 13(31)$ & $10 / 12(83)$ & 0.02 \\
BAEPs & $2 / 13(15)$ & $7 / 10(70)$ & 0.02 \\
Bilateral abnormalities & $0 / 13(0)$ & $5 / 10(50)$ & 0.01 \\
mSEPs & $2 / 13(15)$ & $10 / 11(91)$ & 0.001 \\
Bilateral abnormalities & $1 / 13(7)$ & $7 / 11(64)$ & 0.01 \\
tSEPs & $6 / 12(50)$ & $12 / 12(100)$ & 0.01 \\
Bilateral abnormalities & $3 / 12(25)$ & $11 / 12(92)$ & 0.004 \\
Upper limb MEPs & $2 / 12(17)$ & $11 / 11(100)$ & 0.001 \\
Bilateral abnormalities & $1 / 12(8)$ & $10 / 11(91)$ & 0.001 \\
Lower limb MEPs & $4 / 12(33)$ & $11 / 11(100)$ & 0.004 \\
Bilateral abnormalities & $4 / 12(33)$ & $11 / 11(100)$ & 0.004 \\
\hline
\end{tabular}

${ }^{\star} \chi^{2}$ Test. multiple sclerosis and in one patient $(8 \%)$ with benign multiple sclerosis ( $p<0.001)$.

Eight $(61 \%)$ patients with secondary progressive multiple sclerosis had neurophysiological abnormalities indicative of cervical cord involvement (delayed or absent N13 with normal P11 and/or increased P11-P14 interpeak latencies ${ }^{12}$ ), whereas these occurred in only one $(8 \%)$ patient with benign multiple sclerosis $(p<0.01)$.

\section{Discussion}

The mechanisms underlying the development of disability in multiple sclerosis need to be investigated further to better delineate the natural history of the disease and to correctly monitor the treatments. Brain MRI and multimodal EPs have proved to be excellent for diagnosing multiple sclerosis, ${ }^{891314}$ but their potential roles in monitoring future treatment trials depends exclusively on their ability to parallel clinical manifestations of multiple sclerosis and explain how multiple sclerosis might cause disability. In this study, we compared the MRI and EP findings for two groups of patients with multiple sclerosis, which had sharply different patterns of disease evolution.

\section{EXTENT OF LESIONS}

The main result of this study was the finding that the MRI total lesion load was significantly lower for patients with benign multiple sclerosis than for patients with secondary progressive multiple sclerosis. This result conflicts with those of other studies. ${ }^{1315}$ Technical and methodological considerations are, however, readily apparent to explain these discrepancies. Firstly, these previous studies were performed with machines operating at lower magnetic field strengths $(0 \cdot 15$ or 0.5 Tesla). A correlation between the severity of brain involvement as detected by MRI and the degree of disability was found in cross sectional studies with 1.5 Tesla machines ${ }^{916}$ but not in other studies with less powerful MR scanners. ${ }^{17-18}$ Secondly, none of the previous studies are free from criticism in the selection of patients. In the study by Koopmans et $a l^{3}{ }^{3}$ patients with benign multiple sclerosis were compared with patients with chronic-progressive multiple sclerosis. The chronic-progressive group consisted of patients with both secondary and primary progressive multiple sclerosis. Recent MRI studies ${ }^{12}$ have shown that both the lesion loads and the patterns of evolution of the lesions are very different in patients with primary progressive multiple sclerosis and all other patients with multiple sclerosis. The patients with primary progressive multiple sclerosis seem to have very mild brain involvement. This finding was confirmed clinically by a neuropsychological study that indicated that patients with primary progressive multiple sclerosis rarely have cognitive deficits. ${ }^{19}$ Thompson et al studied patients with benign multiple sclerosis with a duration of the disease about three times longer than that of patients with secondary 
progressive multiple sclerosis. ${ }^{1}$ As remyelinating mechanisms are active from the first stages of development of the lesions, ${ }^{6}$ it is possible that the patients with benign multiple sclerosis evaluated in the study of Thompson et al remained free of disability because the slowness of the pathological process allowed time for remyelination to proceed. Finally, in the study by Filippi et $a l,{ }^{15}$ patients with secondary progressive multiple sclerosis had total and infratentorial lesion volumes greater than the benign group, with an infratentorial lesion volume of $5 \%$ in patients with secondary progressive multiple sclerosis compared with about $2 \%$ in patients with benign multiple sclerosis. These differences, although clinically important, were not statistically significant because of the small number of patients (five with benign multiple sclerosis and six with secondary progressive multiple sclerosis).

About $40 \%$ of patients with benign multiple sclerosis had total lesion loads smaller than a half of the lowest value for patients with secondary progressive multiple sclerosis (figure). This suggests that in many patients with benign multiple sclerosis, the absence of disability is strictly related to the fact that the CNS is relatively spared by the pathological process. Which mechanisms lead to this large reduction in lesion development remain unknown and immunological studies of this group of patients with multiple sclerosis are needed. At this stage, it is possible to suggest that the disease is less active, as shown by Thompson et al, ${ }^{20}$ who found significantly fewer gadolinium enhancing lesions in patients with benign multiple sclerosis than in those with early relapsing-remitting multiple sclerosis. This is also suggested by our data, as only one of five patients with benign multiple sclerosis and mild brain involvement had two gadolinium enhancing lesions of less than 5 $\mathrm{mm}$ in size.

It is also of interest that confluence of the lesions is more often detectable in patients with secondary progressive multiple sclerosis. This finding, which agrees with previous studies, ${ }^{13}$ further supports the presence of a greater cerebral lesion load in these patients than in benign multiple sclerosis, because when confluence is extensive, the actual lesion load is usually underestimated by the method we used. Therefore, it is possible that future studies with volumetric assessment of MRI lesions will show the difference between the two clinical groups better. Furthermore, it is possible that normal appearing white matter might be more severely affected in patients with large confluent lesions. It is well known that there are subtle changes in the normal appearing white matter of patients with multiple sclerosis. ${ }^{21-25}$ Therefore, another possible explanation for the increased disability, at least in those patients with secondary progressive multiple sclerosis and confluent lesions, might be a greater severity of microscopic changes in normal appearing white matter.

On the basis of all these data, there is no doubt that the greater total amount of brain lesions plays an important part in causing the greater degrees of disability in patients with secondary progressive multiple sclerosis than in patients with benign multiple sclerosis. This is not, of course, the direct and unique cause of disability in multiple sclerosis; firstly, because the vast majority of the MRI lesions in our patients were supratentorial, whereas disability, as assessed by EDSS, reflects mainly cerebellar, brainstem, and spinal cord involvement. Secondly, about $60 \%$ of patients with benign multiple sclerosis had total lesion loads overlapping those of patients with secondary multiple sclerosis, and in about $20 \%$ of our patient pairs, patients with benign multiple sclerosis had greater lesion loads than the matched patients with secondary progressive multiple sclerosis.

Therefore, other factors underlying the development of disability in multiple sclerosis must be considered. Our results lend support for at least three other factors: the pattern of distribution of brain lesions, the characteristics of the pathological process, and the extent of spinal cord involvement.

PATTERN OF DISTRIBUTION OF BRAIN LESIONS Some brain regions seem to be involved more often in secondary progressive multiple sclerosis-that is, the periventricular areas (including areas around the fourth ventricle), brainstem, basal ganglia, and corpus callosum. This finding is of the greatest value, as it is obvious that greater involvement of infratentorial structures could be related to more severe disability. Also, it is known that patients with chronic-progressive multiple sclerosis, a group that includes patients with primary and secondary progressive multiple sclerosis, exhibit a decline in cognitive function more often than patients with relapsingremitting multiple sclerosis, ${ }^{26-28}$ a group that also includes patients with benign multiple sclerosis. Therefore, the greater extent of the pathological process detected here in the periventricular regions, basal ganglia, and corpus callosum might be the direct or, more probably as shown by a PET study, ${ }^{29}$ the indirect cause, through cortical diaschisis phenomena, of the mental disability of these patients.

\section{CHARACTERISTICS OF THE PATHOLOGICAL} PROCESS

Recent quantitative $\mathrm{MRI}^{30-32}$ and electron microscopic studies ${ }^{31}$ suggest that chronic lesions in multiple sclerosis may be either "closed" - that is, predominantly cellular with pronounced gliosis, or "open"-that is, with much expanded extracellular space and severe axonal loss. Also, Dousset et $a l,{ }^{25}$ by means of magnetisation transfer imaging, showed highly variable degrees of tissue destruction in multiple sclerosis lesions. There seems to be a greater number of destructive lesions (lesions characterised by more severe axonal loss and demyelination) in patients with secondary progressive multiple sclerosis, causing neurological deficits with little potential for recovery. ${ }^{51533}$ A greater number of such lesions should result in greater parenchymal atrophy, 
potentially detectable by MRI. Our patients with secondary progressive multiple sclerosis did indeed have a higher median score for atrophy of the infratentorial regions. This finding fits perfectly with the demonstration that infratentorial regions of patients with secondary progressive multiple sclerosis are also characterised by a greater extent of lesions. Nevertheless, standard T2 weighted scans give little information about pathological severity, whereas multimodal EPs are, of course, of greater interest.

In chronic multiple sclerosis lesions, both demyelination and axonal loss may cause abnormalities of the EPs. Segmental demyelination can cause variable combinations of conduction block, slowing of nervous conduction, and desynchronisation of the travelling impulse. There is good evidence that conduction block and desynchronisation are more important than slowing for producing the major symptoms of multiple sclerosis. ${ }^{5}$ These conditions are also reflected by different EP abnormalities. In fact, the slowing of conduction delays the EP components, whereas conduction block and impulse desynchronization decrease amplitude and, possibly, lead to the absence of the EP waves. This finding is also typical of processes characterised by axonal loss. ${ }^{34}$ Therefore, EP abnormalities reflect the nature of the underlying lesion pathology more specifically than standard MRI sequences, which can only detect modifications of water content of the tissues. The water content may be due to oedema, demyelination, gliosis, and axonal loss, which could have very different effects on the disability.

In this study the frequencies were significantly higher and the severities of EP abnormalities significantly greater in patients with secondary progressive multiple sclerosis. This might be related to the total extent of the lesions (multiple lesions involving by chance the same pathways could cause EP abnormalities more easily), but also might be an indirect index of a more aggressive pathological process. This hypothesis is supported by a quantitative MRI study ${ }^{15}$ that showed greater proportions of chronic lesions with T2 magnetisation decay characteristics of "biexponentiality" (index of axonal degeneration ${ }^{31}$ ) in patients with secondary progressive multiple sclerosis than in those with benign multiple sclerosis and by an MTI study that found areas of low MT ratio more often in patients with clinically severe multiple sclerosis (primary and secondary progressive) than in patients with benign multiple sclerosis. ${ }^{33}$

Finally, motor, somatosensory, and visual EPs explore directly CNS pathways of crucial importance in determining disability when affected by multiple sclerosis lesions. Furthermore, BAEPs are generated by structures present diffusely and interconnected variously in the brainstem, giving indirect information about the actual activity of this functionally vital region. Therefore, the finding that EP abnormalities are more often detectable and are more severe in patients with secondary progressive multiple sclerosis is of the greatest importance when considering the causes of disability in multiple sclerosis.

It is also of interest that the number of enhancing lesions in patients with secondary multiple sclerosis was two-fold higher than in those with benign multiple sclerosis. Also, only $20 \%$ of these lesions in patients with benign multiple sclerosis had diameters larger than $5 \mathrm{~mm}$, whereas enhancing lesions larger than $5 \mathrm{~mm}$ were $50 \%$ of the total in patients with secondary progressive multiple sclerosis. These differences were not statistically significant because of the small number of patients with enhancing lesions, due to the inclusion criteria for patients. Therefore, persistent activity in the development of new lesions in more disabled patients even many years after the onset of the disease must be considered and evaluated in further longitudinal studies. It seems likely that frequent recurrent episodes of inflammation and demyelination, especially if located in the same structures or along the same pathways, could well lead to permanent neurological deficits.

Finally, all the enhancing lesions in patients with benign multiple sclerosis were detected in the five patients with the greatest lesion loads. Moreover, three of these patients had had at least one relapse in the year preceding the inclusion, with an increase of residual disability of 1 point in the EDSS. These data, in agreement with a previous report investigating the relation between the numbers and the areas of enhancing lesions and the onset and continuation of clinical worsening, ${ }^{35}$ suggest that these patients, defined as having benign multiple sclerosis according to the classic criteria based on the duration of the disease and the present disability, might be entering the secondary progressive phase of the disease. If this hypothesis is confirmed in the ongoing follow up, the absence of recent relapses should be added to the definition of benign multiple sclerosis.

\section{EXTENT OF SPINAL CORD INVOLVEMENT}

Recent MRI studies ${ }^{236}{ }^{37}$ failed to show different degrees of spinal cord involvement in patients with multiple sclerosis with different clinical manifestations of the disease, perhaps because of partial volume effects related to the small size of the lesions in the spinal cord. Our study also showed a greater involvement of the cervical spinal cord in patients with secondary progressive multiple sclerosis.

Damage to this CNS region is, of course, crucial in determining disability, as many CNS pathways are packed together in a small space and even small lesions can cause pronounced dysfunction. Also, the significantly higher frequencies of abnormalities of both tSEPs and lower limb MEPs (which were also more often bilateral) in patients with secondary progressive multiple sclerosis also suggest more severe and extensive spinal cord damage in patients with secondary progressive multiple sclerosis.

In conclusion, the results of this study indicate that the extent and severity of the 
pathological process, as detected by MRI and multimodal EPs, are strictly related to the severity of disability in patients with benign and secondary progressive multiple sclerosis. The ongoing follow up of these patients will provide further information about the relation between changes in disability and changes in the instrumental findings in multiple sclerosis, which should be relevant for planning future clinical trials.

We thank Dr David H Miller (NMR Research Group, Institute of Neurology, London, UK) for critical comments on the manuscript. We also thank Mr Clodoaldo Pereira and Mrs Paola Elia for their skilful technical assistance.

1 Thompson AJ, Kermode AG, MacManus DG, et al. Patterns of disease activity in multiple sclerosis: clinical and magnetic resonance imaging study. $B M F 1990 ; 300$ : 631-4.

2 Thompson AJ, Kermode AG, Wicks D, et al. Major differences in the dynamics of primary and secondary progressive multiple sclerosis. Ann Neurol 1991;29: 53-62.

3 Koopmans RA, Li DKB, Grochowski E, et al. Benign versus chronic progressive multiple sclerosis: magnetic resonance imaging features. Ann Neurol 1989;25:74-81.

4 Kermode AG, Thompson AJ, Tofts P, et al. Breakdown of blood-brain-barrier precedes symptoms and other MRI signs of new lesions in multiple sclerosis: pathogenetic and clinical implications. Brain 1990;113:1477-89.

5 McDonald WI, Miller DH, Barnes D. The pathological evolution of multiple sclerosis. Neuropathology and evolution of multiple sclerosis. Neur
Applied Neurobiology 1992;18:319-34.

6 Prineas JW, Barnard RO, Kwon EE, et al. Multiple sclerosis: remyelination of nascent lesions. Ann Neurol 1993 33:137-51.

7 Farlow M, Markand NO, Edwards M, et al. Multiple sclerosis: magnetic resonance imaging, evoked responses and spinal fluid electrophoresis. Neurology 1986;36: 828-31.

8 Giesser B, Kurtzberg D, Vaughan G, et al. Trimodal evoked potentials compared with magnetic resonance imaging in the diagnosis of multiple sclerosis. Arch Neurol 1987;44:281-4.

9 Comi G, Martinelli V, Medaglini S, et al. Correlation between multimodal evoked potentials and MRI in multiple sclerosis. $₹$ Neurol 1989;236:4-8.

10 Poser CM, Paty DW, McDonald WI, et al. New diagnostic criteria for multiple sclerosis: guidelines for research criteria for multiple sclerosis: guidelimes

11 Kurtzke JF. Rating neurologic impairment in multiple sclerosis: an expanded disability status scale (EDSS) Neurology 1983:33:1444-52.

12 Mauguiere F, Ibanez V. The dissociation of early SEP components in lesions of the cervico-medullary junction A clue for routine interpretation of abnormal cervical responses to median nerve stimulation. Electroencephalog Clin Neurophysiol 1985;62:406-20.

13 Paty DW, Oger JF, Kastrukoff LF, et al. MRI in the diagnosis of multiple sclerosis: a prospective study with comparison of clinical evaluation, EPs, oligoclonal banding and CT. Neurology 1988;38:180-5.

14 Ormerod IEC, Miller DH, McDonald WI, et al. The role of NMR imaging in the assessment of multiple and isolated neurological lesions. Brain 1987;110:1579-616.

15 Filippi M, Barker GJ, Horsfield MA, et al. A quantitative brain MRI study of benign and secondary progressive multiple sclerosis. 7 Neurol 1994;241:246-51.

16 Truyen L, Gheuens J, van de Vyver FL, et al. Improved correlation of magnetic resonance imaging with clinical status in multiple sclerosis by use of an extensive standardized imaging-protocol. F Neurol Sci 1990;96: 173-82.

17 Palo J, Ketonen L, Wickstrom J. A follow-up study of very low field MRI findings and clinical course in multiple sclerosis. F Neurol Sci 1988;84:177-87.

18 Baumhefner RW, Tourtellotte WW, Syndulko K, et al. Quantitative multiple sclerosis plaque assessment with magnetic resonance imaging. Its correlation with clinical parameters, evoked potentials, and intra-blood-brain barrier IgG synthesis. Arch Neurol 1990;47:19-26.

19 Filippi M, Alberoni M, Martinelli V, et al. The influence of clinical variables on neuropsychological performance in multiple sclerosis. Eur Neurol 1995 (in press)

20 Thompson AJ, Miller D, Youl B, et al. Serial gadoliniumenhanced MRI in relapsing-remitting multiple sclerosis of varying disease duration. Neurology 1992;42:60-3.

21 Adams CMW. Pathology of multiple sclerosis: progression of the lesion. Br Med Bull 1977;33:15-20.

22 Allen IV, McKeown SR. A histological, histochemical and biochemical study of the macroscopically normal white biochemical study of the macroscopically normal white

23 Miller DH, Johnson G, Tofts PS, et al. Precise relaxation time measurements of normal-appearing white matter in inflammatory central nervous system disease. Magn Reson Med 1989;11:331-6.

24 Haughton VM, Yetkin FZ, Rao SM, et al. Quantitative $M R$ in the diagnosis of multiple sclerosis. Magn Reson Med 1992;26:71-8.

25 Dousset V, Grossman RI, Ramer KN, et al. Experimental allergic encephalomyelitis and multiple sclerosis: lesion characterization with magnetization transfer imaging. Radiology 1992;182:483-91.

26 Heaton RK, Nelson LM, Thompson DS, et al. Neuropsychological findings in relapsing-remitting and chronic progressive multiple sclerosis. F Consult Clin Phronic progressive mul 1985;53:103-10.

27 Beatty WW, Goodkin DE, Monson N, et al. Cognitive disturbances in patients with relapsing-remitting disturbances in patients with relapsing-rem
multiple sclerosis. Arch Neurol 1989;46:1 11 13-9.

28 Feinstein A, Kartsounis LD, Miller DH, et al. Clinically isolated lesions of the type seen in multiple sclerosis: a cognitive, psychiatric, and MRI follow-up study. F Neurol Neurosurg Psychiatry 1992;55:869-76.

29 Comi G, Perani D, Martinelli V, et al. Cognitive function impairment in multiple sclerosis: a 18F-FDG and PET study. In: H Wietholter, ed. Current concepts in multiple sclerosis. Amsterdam: Elsevier Science Publishers 1991: $55-60$.

30 Larsson HBW, Frederiksen J, Kjaer L, et al. In vivo determination of $T_{1}$ and $T_{2}$ in the brain of patients with severe but stable multiple sclerosis. Magn Reson Med 1988;7: 43-55.

31 Barnes D, Munro PMG, Youl BD, et al. The longstanding MS lesion. A quantitative MRI and electron microscopic MS lesion. A quantitative MRI and
study. Brain 1991;114:1271-80.

32 Newcombe J, Hawkins CP, Henderson CL, et al. Histopathology of multiple sclerosis lesions detected by magnetic resonance imaging in unfixed postmortem central nervous system tissue. Brain 1991;114:1013-23.

33 Gass A, Barker GJ, Kidd D, et al. Correlation of magnetisation transfer ratio with clinical disability in multiple sclerosis. Ann Neurol 1994;36:62-7.

34 Livingstone IR, Mastaglia FL, Edis R, Howe WJ. Visual involvement in Friedreich's ataxia and hereditary spastic ataxia. Arch Neurol 1981;38:75-9.

35 Smith ME, Stone LA, Albert PS, et al. Clinical worsening in multiple sclerosis is associated with increased frequency and area of gadopentetate dimeglumine-enhancing magnetic resonance imaging lesions. Ann Neurol 1993; 33:480-9.

36 Wiebe S, Lee DH, Karlik SJ, et al. Serial cranial and spinal cord magnetic resonance imaging in multiple sclerosis. cord magnetic resonance imagr

37 Kidd D, Thorpe JW, Thompson AJ, et al. Spinal cord MRI using multi-array coils and fast spin echo. II. Findings in multiple sclerosis. Neurology 1993;43:2632-7. 